\title{
The Emerging Synergy between Radioembolization, Systemic Chemotherapy, and Liver Surgery in Metastatic Colorectal Cancer
}

\author{
a report by \\ Harpreet S Wasan, MD \\ Consultant in Medical Oncology and Honorary Senior Lecturer, Imperial College London, and Department of Cancer Medicine, Hammersmith Hospital
}

DOI: 10.17925/EOH.2007.0.1.52

Colorectal cancer is one of the most common malignancies and a leading cause of death. It is estimated that during 2006 there were 412,900 new cases of colorectal carcinoma diagnosed in Europe and 207,500 deaths. The five-year survival of patients with metastatic disease is less than $7 \%$. Hepatic metastases are present in 15-25\% of patients at the time of diagnosis of colorectal cancer, and another 15-20\% will develop metachronous liver metastases within five years following resection of the primary tumor. ${ }^{2}$ Progression of liver disease also leads to significant clinical morbidity, which is frequently correlated with overall survival.' The earlier identification of liver metastases should improve the management and survival of patients with colorectal cancer.'

Unfortunately, only a small proportion-between 10 and 15\%-of patients with liver metastases only are primary candidates for potentially curative liver resection, since surgery is limited to those with a good performance status, and those who fall within 'limited disease criteria' (generally four or fewer tumors in one lobe of $<4 \mathrm{~cm}$ each, not involving major vascular structures). Of those who do have a complete surgical resection undertaken with curative intent, the five-year survival rate is $30-40 \%$, with at least two-thirds of patients developing recurrent liver metastases within two years after surgery. ${ }^{4}$ The majority of patients with liver metastases are initially not candidates for liver resection and are managed with systemic chemotherapy; there have been major palliative advances in this field over the last 10 years.

Until the mid-1990s the only available drug — which had limited activity in metastatic colorectal cancer-was fluorouracil (5FU). The last decade has seen a rapid evolution of systemic chemotherapies for metastatic colorectal cancer with the development of new cytotoxic agents such as

Harpreet S Wasan, MD, leads the gastrointestinal clinical research program at Hammersmith Hospital, Imperial College London. He is on the UK National Cancer Research Institute Steering Committee of both the advanced colorectal cancer and upper gastrointestinal groups, as well as being the inaugural Chair of the West London Cancer Network. Dr Wasan is involved in the trial management of many colorectal carcinoma studies (CRC)-including one of the largest metastatic colorectal cancer studies in the world (COIN study) - and is the chief investigator in its sub-study (COIN-B), as well as a co-investigator on the largest prospective study in chemotherapy in the elderly (FOCUS-2), which reports in 2007. He is on the European working party on CRC liver metastases guidance. In upper gastrointestinal cancer he is co-principal investigator (PI) and PI in the UK NRCN trials portfolio in advanced biliary cancer and cancer of unknown primary, with the latter two designed to be the largest studies of their kind in the world. He is a Fellow of the Royal College of Physicians and the oncology advisor to the UK Drugs and Therapeutics Bulletin, among many other national consultative roles. Dr Wasan graduated from University College London Medical school in 1985 and trained in gastroenterology; he specializes in gastrointestinal medical oncology. He has also spent three years in basic science at the ICRF (now CRUK) working on the APC gene and intestinal crypt biology. oxaliplatin and irinotecan; oral analogs (Capecitabine; Xeloda ${ }^{\circledR}$ and UFT-tegafur); and targeted biologicals such as bevacizumab (a monoclonal antibody against vascular endothelial growth factor) and cetuximab and panitumumab (monoclonal antibodies against the epidermal growth factor receptor type-1).

Combined with 5FU plus leucovorin (LV), irinotecan-based (FOLFIRI) and oxaliplatin-based (FOLFOX) regimens have evolved as standard first- and second-line treatments for metastatic colorectal cancer (see Table 1). More recently, phase $\| / / I I$ clinical trials have shown that the addition of bevacizumab increases the activity of 5FU/LV, irinotecan combinations, ${ }^{5}$ and oxaliplatin-based (FOLFOX) regimens. ${ }^{6}$ Recent trials (XELOXA/NO16966 and CRYSTAL) have suggested that biologicals may not provide the level of added benefit that was originally hoped for.?

These important new advances in biologic and cytotoxic agents have resulted in a significant prolonging of median survival time from approximately six months with 5FU-containing chemotherapy regimens ${ }^{8}$ to an excess of 20 months in some studies. ${ }^{5,9,10}$ Yet, despite these valuable gains, metastatic colorectal cancer is almost always fatal, with up to $90 \%$ of patients dying of liver failure caused by local effects of hepatic tumors. In high-risk patients with non-resectable and chemo-refractive liver tumors, therapies should be considered palliative, with the primary aim of treatment being to achieve a maximum reduction in tumor cell burden and improvements in progressionfree survival and quality of life, as well as improving overall survival.

External-beam radiation therapy (EBRT) is a cornerstone of curative and palliative therapy in nearly all malignancies, but has not been applied with much success to hepatic disease due to the low tolerance of the organ to radiation compared with tumor. Significant technological advances in radiation treatment planning and delivery — which may benefit an increasing number of patients with liver metastases-have led to improved tumordirected radiotherapy approaches, such as:

- 3-D radiotherapy;

- intensity-modulated radiotherapy; and

- stereotactic radiotherapy.

However, the key limitation of this treatment is the tolerance of normal liver parenchyma to radiation. The maximum acceptable dose to the whole liver is $35 \mathrm{~Gy}$, which is far below the required dose to destroy adenocarcinoma metastases, estimated at 70Gy or above (for monotherapy) or at 50Gy or above when concurrent chemotherapy is given. An alternative approach is the implantation of radiation sources into the tumor (brachytherapy). 
One form of brachytherapy is radioembolization, or selective internal radiation therapy (SIRT), which has been successfully utilized for the treatment of liver metastases and primary liver tumors. This procedure delivers radiation particles intravascularly, and takes advantage of the differential blood supply to tumors and normal parenchyma in order to ensure tumor-directed therapy. Eighty percent of the blood supply to the liver is via the portal vein, whereas almost all of the blood supply to the tumors is via the hepatic artery. By using the hepatic artery to inject embolic particles, multiple sites of disease within the liver can be selectively irradiated in a single procedure. The radiation is delivered by SIR-Spheres ${ }^{\circledR}$ (Sirtex Medical, Australia), which are biocompatible microspheres containing the pure beta-emitter radionuclide yttrium-90, which decays to stable zirconium-90 with an average half-life of 2.67 days (64.2 hours). The microspheres, with a mean diameter of $32 \mu \mathrm{m}$, lodge within malignant microvasculature where they deliver high localized doses of ionizing radiation (100-3,000Gy) to tumors and the neovascular supply of the tumor while delivering safe levels to the normal tissue (mean penetration of radiation into tissues is $2.4 \mathrm{~mm}) .{ }^{11}$ Notably, the size of the microspheres means that they cannot pass into the capillary bed.

This loco-regional approach has been used for many years to deliver chemoembolization to hepatic malignancies, but such treatments have not provided any evidence of survival benefits in metastatic colorectal cancer ( $m C R C)$. In contrast, there is a growing body of evidence demonstrating the efficacy of radioembolization using SIR-Spheres in improving the treatmentrelated response, time to disease progression, and survival either in combination with systemic chemotherapy compared with chemotherapy alone $^{12,13}$ or as a monotherapy. ${ }^{14}$ The evidence shows that responders appreciate a longer survival with little, if any, detrimental impact of treatment on quality of life.

The Clinical Evidence for Radioembolization with SIR-Spheres In parallel with the evolution of systemic chemotherapy, a series of randomized, controlled trials has been conducted combining SIR-Spheres initially with hepatic artery chemotherapy then systemic chemotherapy, as well as in single-arm dose-escalation studies. A summary of the results from these trials is outlined in Table 2.

In one of the early trials, SIR-Spheres were evaluated as a first-line treatment in patients with non-resectable liver-only colon cancer metastases. ${ }^{12}$ Combined with hepatic artery chemotherapy with floxuridine, patients treated with SIR-Spheres showed a significant reduction in tumor volume $(p=0.03)$ and increase in time to progression (15.9 versus 9.7 months; $\mathrm{p}=0.001$ ) compared with those receiving hepatic artery chemotherapy alone (see Table 2). The one-, two, three-, and five-year survival for patients receiving SIR-Spheres was $72 \%, 39 \%, 17 \%$, and 3.5\%, respectively, compared with $68 \%, 29 \%, 6.5 \%$, and $0 \%$, respectively, for hepatic artery chemoembolization alone. However, the study was halted prematurely and not powered adequately to show survival advantage. ${ }^{12}$ Nevertheless, the study was important in demonstrating the efficacy and tolerability of SIR-Spheres, and cox regression analysis suggested a benefit for patients treated with SIR-Spheres surviving beyond 15 months ( $p=0.06$ ). Moreover, the addition of SIR-Spheres microspheres to hepatic artery chemotherapy was found to have no major adverse impact on quality of life, with both treatment groups demonstrating some improvement in quality of life.

It is apparent from clinical reports that one of the principle determinants of
Table 1: Investigational First-line Treatments for Metastatic Colorectal Cancer

\begin{tabular}{ll} 
FOLFOX & Oxaliplatin + fluorouracil + leucovorin \\
\hline XELOX & Oxaliplatin + capecitabine \\
\hline FOLFIRI & Irinotecan + fluorouracil + leucovorin \\
\hline FOLFOXIRI & Oxaliplatin + irinotecan + fluorouracil + leucovorin \\
\hline $\begin{array}{l}\text { Bevacizumab + fluorouracil/ } \\
\text { leucovorin }\end{array}$ & Bevacizumab + fluorouracil + leucovorin \\
\hline Bevacizumab + FOLFIRI & Bevacizumab + irinotecan + fluorouracil + leucovorin \\
\hline Bevacizumab + FOLFOX & Bevacizumab + oxaliplatin + fluorouracil +leucovorin \\
\hline
\end{tabular}

Figure 1: Kaplan-Meier Survival Curve Following First-line Treatment SIR-Spheres plus 5FU/LV or 5FU/LV Alone in 21 Patients with Metastatic Colorectal Cancer ${ }^{12}$

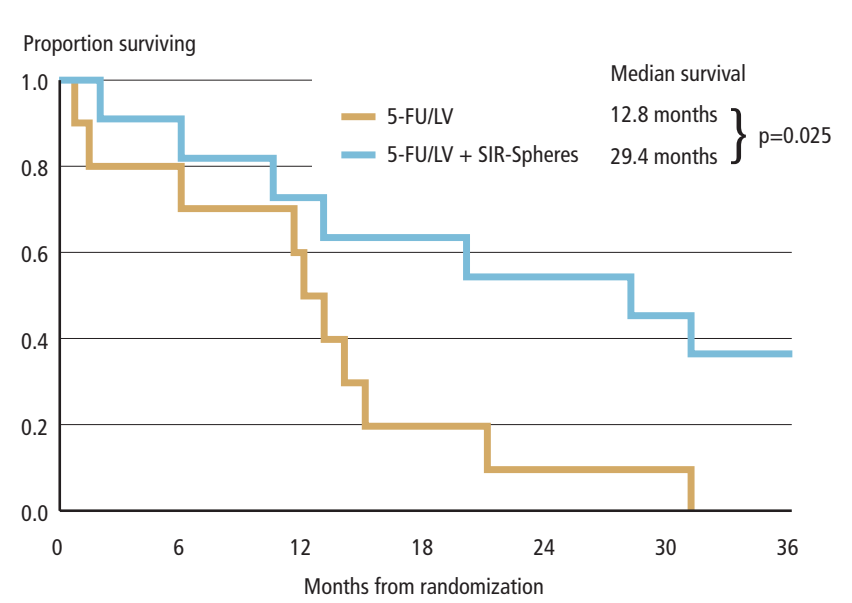

Figure 2: Kaplan-Meier Survival Curve from Day of Treatment to Censure or Death in 208 Patients with Chemotherapy-refractory Disease Treated with SIR-Spheres

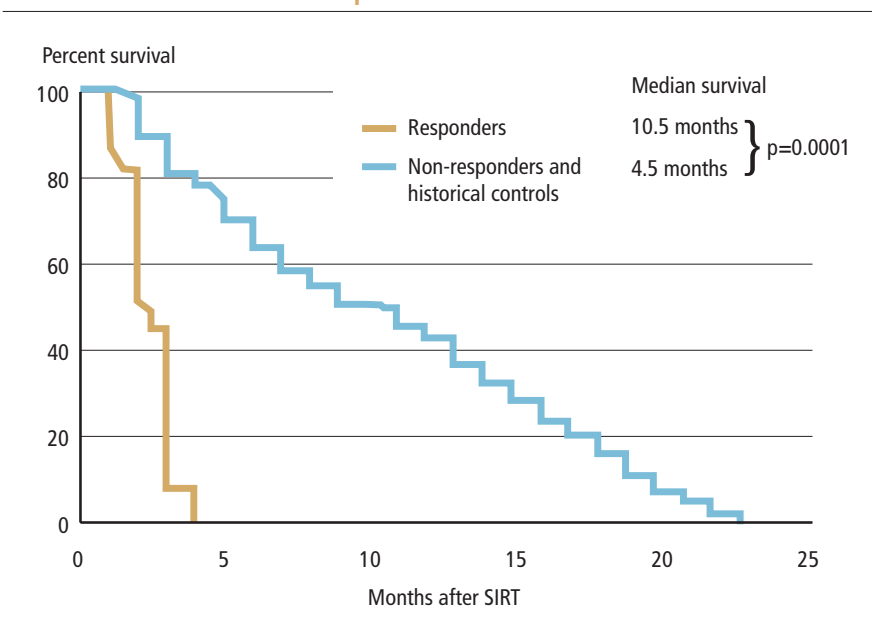

survival after radioembolization is the presence or subsequent development of clinically relevant extrahepatic disease. The experience of investigators indicates that median survival times are reduced in patients who either have significant extrahepatic disease prior to treatment or who develop such disease within six months of radioembolization compared with those who did not ( 8.3 versus 12.6 months; $p<0.001$ ). ${ }^{14}$ These results suggest that localized treatment with SIR-Spheres should be combined with systemic chemotherapy in order to achieve the maximum survival benefit. 
Figure 3: Comparison of the Objective Response Rates from Reported Studies of Chemotherapy Regimens either with or without Biological Agents, or with or without Radioembolization Using SIR-Spheres ${ }^{11,12,15,40-44}$

\section{Chemotherapy \pm biologicals}

Chemotherapy \pm SIRT

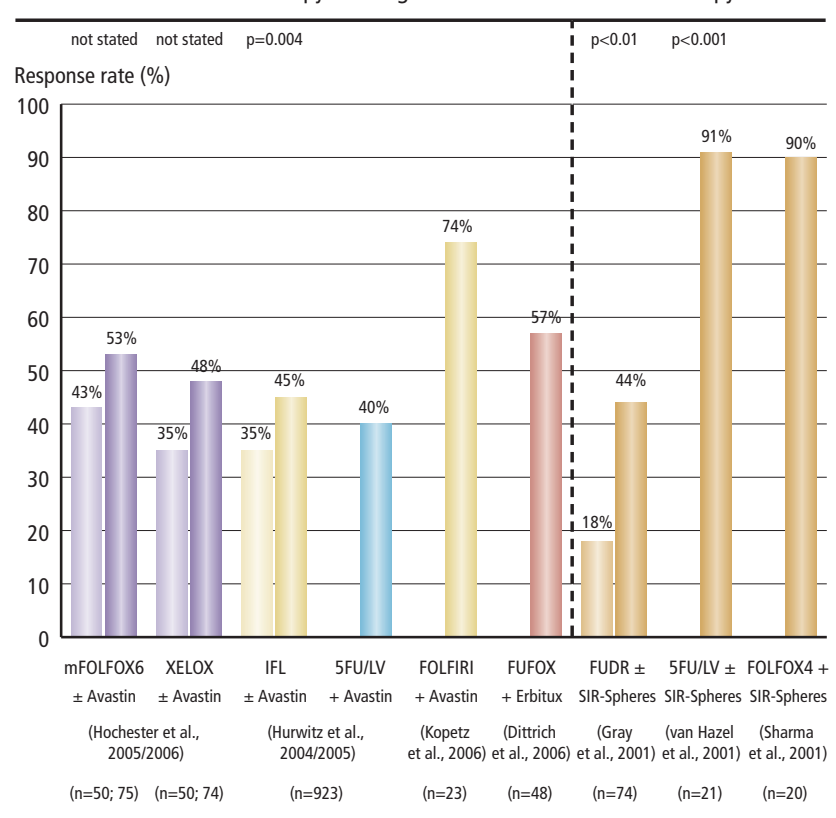

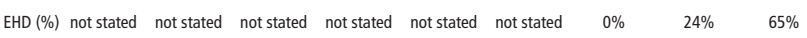

Figure 4: Comparison of the Median Time to Progression or Progression-free Survival from Reported Studies of Chemotherapy Regimens either with or without Biological Agents, or with or without Radioembolization Using SIR-Spheres s, $11,12,15,42-44^{-4}$

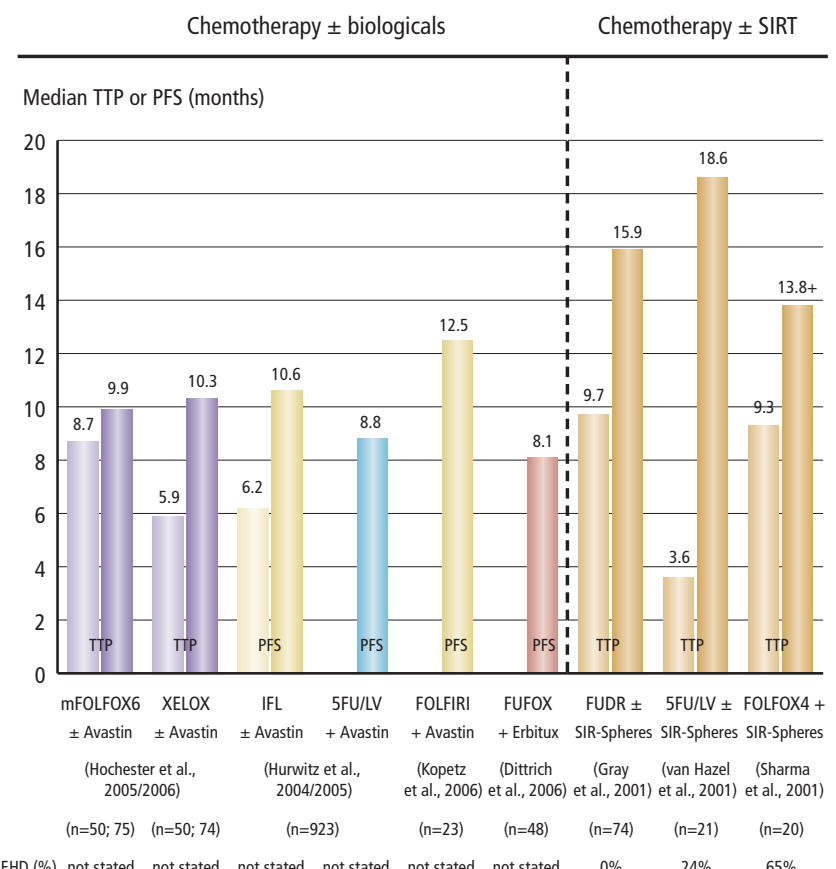

EHD (\%) not stated not stated not stated not stated not stated not stated $\quad 0 \% \quad 24 \% \quad 65 \%$

Subsequently, a randomized, controlled trial combining SIR-Spheres with 5FU/LV systemic chemotherapy was conducted in 21 patients with previously untreated advanced colorectal liver metastases, with or without extrahepatic metastases..$^{13}$ The study found that the addition of a single administration of SIR-Spheres to a regimen of 5FU/LV chemotherapy significantly increased both time to disease progression (18.6 versus 3.6 months; $p<0.0005$ ) and median survival by more than 16 months (29.4 versus 12.8 months; $p=0.02$ ) compared with system chemotherapy alone (see Figure 1). ${ }^{12}$

Adoption of radioembolization in most clinics started conservatively as salvage therapy. A retrospective analysis was conducted in 208 patients with unresectable liver-predominant metastases from colorectal cancer that were refractory to oxaliplatin-based (FOLFOX) regimens, irinotecan-based (FOLFIRI) regimens, or third-line chemotherapy and were not eligible for liver-directed therapy (including radiofrequency ablation (RFA), transarterial chemo-embolization (TACE), resection, intensity-modulated radiotherapy (IMRT), and stereotactic radiotherapy). After a single dose of SIR-Spheres, patients were followed up for a median of 13 months (range 1-42 months). In total, positron emission tomography (PET) scans showed response in 176 of 208 patients (85\%). The study showed a significant survival benefit in the majority of patients responding to SIR-Spheres (see Figure 2). Researchers found that if patients did not experience a measurable response (carcinoembryonic antigen (CEA), PET, or computed tomography (CT) scan) by six weeks after treatment, their survival was short (median 4.5 months) compared with responders, who achieved an appreciably longer survival (median 10.5 months; $\mathrm{p}=0.0001$ ). ${ }^{13}$

Although the early randomized, controlled trials involved regimens that are now considered outdated and were inadequately sized to show a convincing survival benefit, the potential was sufficient to encourage further assessment of the safety and tolerability and dosing of SIR-Spheres combined with modern agents. Phase I studies of SIR-Spheres + FOLFOX and SIR-Spheres + irinotecan have shown impressive response rates and progression-free survival. ${ }^{16-19}$

Sharma and colleagues treated newly diagnosed patients with FOLFOX4 and one application of SIR-Spheres during the first week of chemotherapy. A partial response by CT scan using Response Evaluation Criteria in Solid Tumors (RECIST) criteria was confirmed in 18 of 20 patients (90\%), with stable disease in the remaining patients and no progressive disease. ${ }^{16}$ After 12 cycles of chemotherapy, three patients were down-staged and two of these patients were surgically resected. Time to progression was 9.3 months (range 3.9-30.7 months) in the whole patient group and 14.2 months (range 6.4-24.9 months) in a subset of seven patients with no extrahepatic metastases at study entry. ${ }^{16}$ Although the patient numbers are small, these results compare favorably with trial data for FOLFOX4 chemotherapy alone in this patient group. ${ }^{20,21}$ In fact, the clinical outcomes of radioembolization in combination with first-line chemotherapy regimens compare favorably with those from current chemotherapy regimens with or without the addition of new biological agents (see Figures 4 and 5).

Van Hazel and Goldstein also tested the efficacy and safety of SIR-Spheres plus second-line chemotherapy with irinotecan. ${ }^{17-19}$ Over a third of the 25 patients included in the study had failed up to three previous lines of chemotherapy and $60 \%$ of patients had failed prior oxaliplatin treatment. The study showed that a single dose of SIR-Spheres in combination with irinotecan was associated with an acceptable toxicity profile. ${ }^{17}$ An overall response was achieved in $48 \%$ of patients and disease control in $87 \%$. Median time to progressive disease was 9.2 months in the liver and 6.0 months at any site including the liver. Median overall survival was 
Table 2: Overview of Clinical Study Results

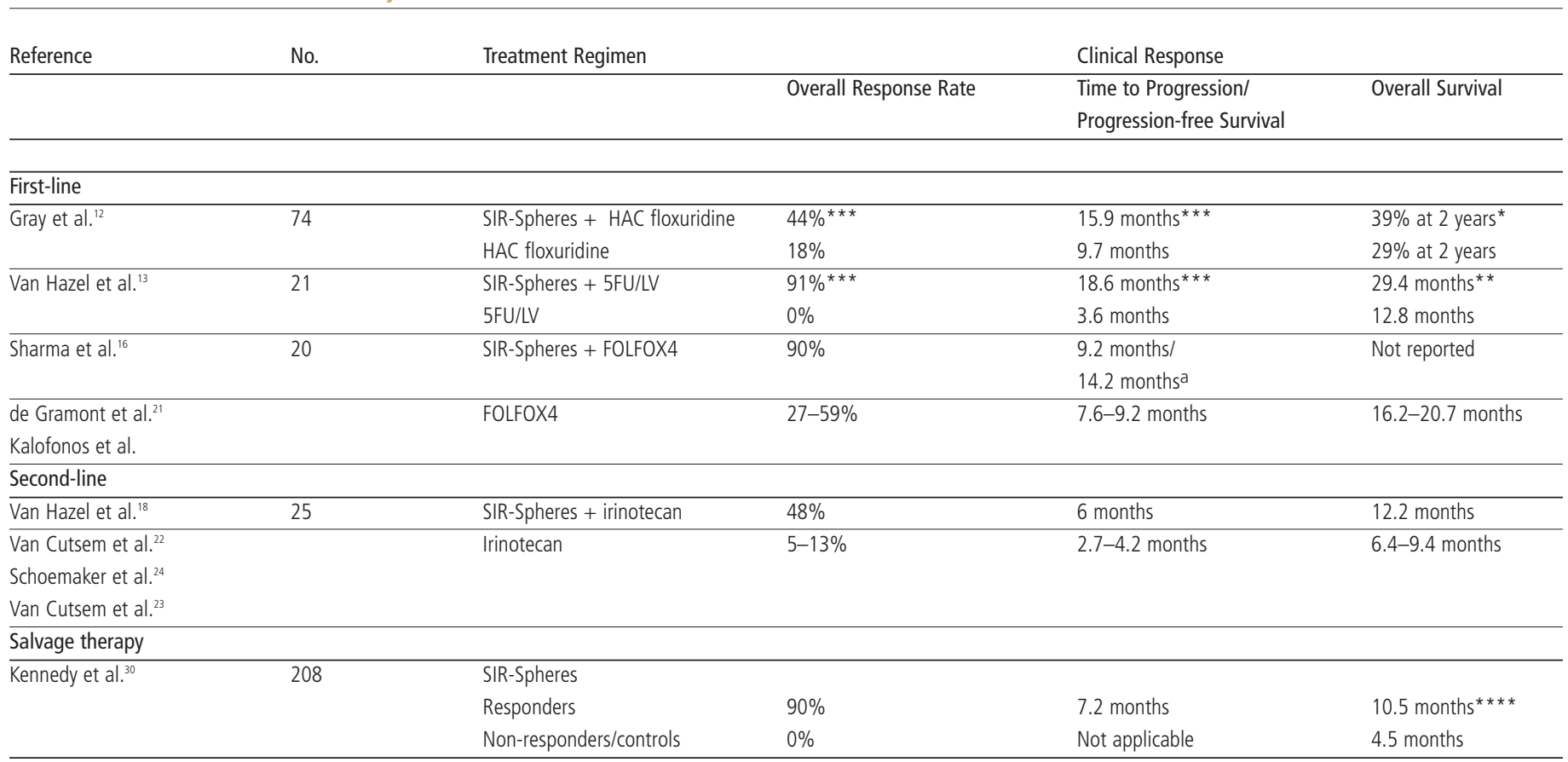

SIR-Spheres for the first- and second-line treatment or salvage therapy of metastatic colorectal cancer.

${ }^{*} p=0.06 ;{ }^{* *} p \leq 0.05 ;{ }^{* *} p \leq 0.001 ;{ }^{* * *} p=0.0001$ SIR-Spheres versus active control.

a Patients with liver-only disease; $H A C=$ hepatic artery infusion; $5 F U=$ fluorouracil; $L V=$ leucovorin.

12.2 months (range 2.8-42.3 months). ${ }^{19}$ Again, these results compare favorably with studies on irinotecan alone in second-line therapy. ${ }^{22-24}$

\section{Radioembolization in the Continuum of Care}

In summary, the evidence indicates a clear synergy between radioembolization with SIR-Spheres and systemic chemotherapy, extending survival and progression-free survival and improving response rates as a firstand second-line therapy, or as a salvage monotherapy in patients with chemorefractory disease. Furthermore, radioembolization with SIR-Spheres appears to be well tolerated and maintains quality of life in patients with unresectable tumors. ${ }^{12}$ The relative ease of administration means that treatment can be delivered on an outpatient basis, or involving one night's hospitalization in the majority of patients.

SIR-Spheres used in conjunction with modern systemic chemotherapies are appropriate both to avoid extrahepatic progression and to prolong the local effects of radioembolization. While the evidence supports the potential of radioembolization using SIR-Spheres to provide substantial clinical benefit for patients with liver-predominant colorectal metastases at various points in the continuum of care-from first-line setting through to salvage therapy-the benefits in reducing tumor burden appear to be greatest early on when the liver vasculature is also less compromised. Moreover, because the liver is the major initial site of metastases, ${ }^{25}$ improved early responses at this site may improve overall survival. With the availability of more effective systemic chemotherapies, strategies are now being developed to resect what in the near past was regarded as 'unresectable' disease. The evidence indicates that this may well be a realistic goal in up to $25 \%$ of patients with metastases confined to the liver who are treated with chemotherapy alone. For these patients, the curative potential of treatment compared with classical palliative end-points of response or progression-free survival becomes a real
Figure 5: Comparison of the Median Overall Survival from Reported Studies of Chemotherapy Regimens either with or without Biological Agents, or with or without Radioembolisation Using SIR-Spheres ${ }^{4,11,12,15,42-44}$

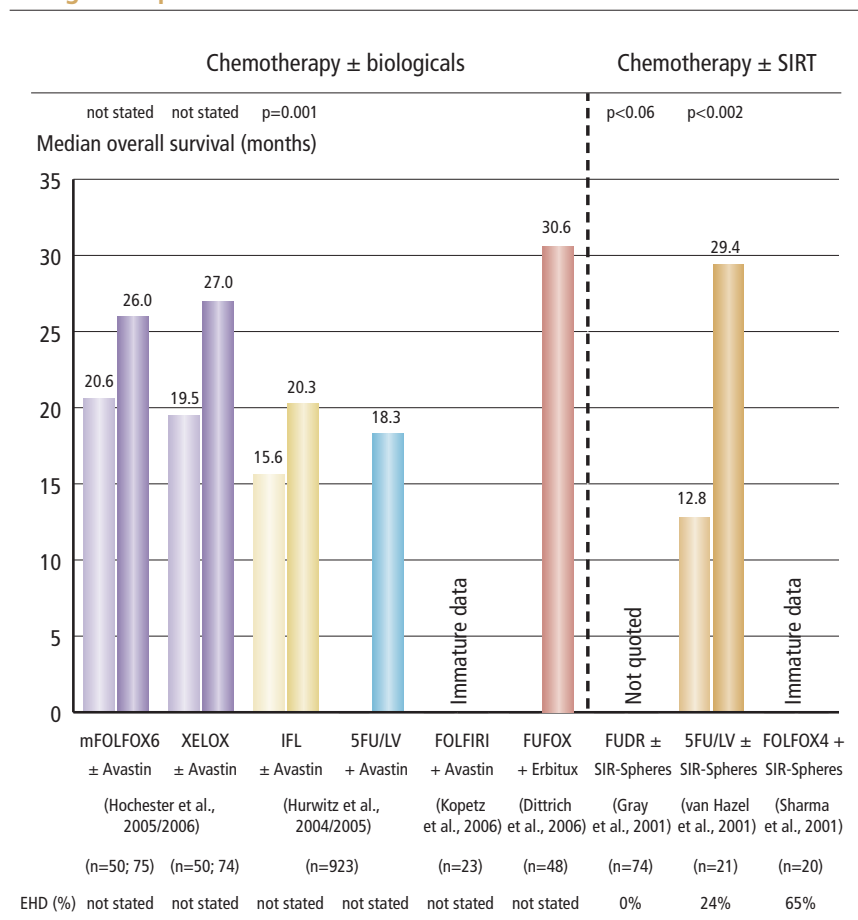

possibility. In a retrospective analysis of published studies, Folprecht and colleagues $^{26}$ found a significant correlation between response rates and the resection rate in studies with patients with isolated liver metastases $(p=0.002)$. It is increasingly likely that radioembolization will be adopted to 
augment the regional response of systemic chemotherapies in the liver and increase the number of patients who are candidates for colorectal liver metastases resection. Application of an innovative multidisciplinary approach that integrates advanced surgical techniques with new drug treatments and therapeutic approaches (such as radioembolization and local ablative therapy) will allow the benefits of curative hepatic resection to be extended to a broader group of patients. However, the optimal management of patients with colorectal liver metastases can be provided only by close co-operation with various specialists (comprising a liver surgeon, oncologist, hepatologist, interventional radiologist, nuclear medicine physician, and histopathologist) who have an in-depth understanding of the latest diagnostic and therapeutic options.
Radioembolization 'Comes of Age"

An international clinical program is now ongoing evaluating radioembolization using SIR-Spheres in phase II/III studies in combination with the newest and most effective chemotherapy agents for colorectal cancer (such as FOLFOX) in the SIRFLOX study and biologicals such as bevacizumab (concurrently administered with either FOLFOX6 or FOLFIRI) in the FAST study, and cetuximab combined with irinotecan in another phase I//II study. Finally, there is increasing evidence that the role of radioembolization is not limited to colorectal liver metastases, since significant clinical benefits have also been reported in liver metastases from breast cancer, ${ }^{27}$ neuroendocrine tumors, ${ }^{28-30}$ and other cancers, ${ }^{31-34}$ as well as in primary liver cancers such as hepatocellular carcinoma ${ }^{35-39}$ and nodular cholangiocarcinoma. ${ }^{40}$ Research into these other indications is also ongoing.
1. Ferlay J, Autier $\mathrm{P}$, Boniol $\mathrm{M}$, et al., Estimates of the cancer incidence and mortality in Europe in 2006, Ann Oncol, 2007;18:581-92.

2. Manfredi S, Lepage C, Hatem C, et al., Epidemiology and management of liver metastases from colorectal cancer, Ann Surg, 2006;244:254-9.

3. McMillan DC, McArdle CS, Epidemiology of colorectal liver metastases, Surg Oncol, 2007;epub: doi:10.1016/j.suronc.2007.04.008. 4. Khatri.

5. Hurwitz $H$, Fehrenbacher L, Novotny $W$, et al., Bevacizumab plus irinotecan, fluorouracil, and leucovorin for metastatic colorectal cancer, N Engl J Med, 2004;350:2335-42.

6. Van Cutsem E, Progress with biological agents in metastatic colorectal cancer leads to many challenges, J Clin Oncol, 2006;24:3325-7.

7. Saltz L, Clarke S, Diaz-Rubio E, et al., Bevacizumab (Bev) in combination with XELOX or FOLFOX4: Updated efficacy results from XELOX-1/ N016966, a randomized phase III trial in first-line metastatic colorectal cancer, ASCO Annual Meeting Proceedings, J Clin Oncol, 2007;25(18S):Abstract 4028.

8. Choti MA, Bulkley GB, Management of hepatic metastases, Liver Transpl Surg, 1999;5:65-80.

9. Falcone A, Ricci S, Brunetti I, et al., Phase III trial of infusional fluorouracil, leucovorin, oxaliplatin, and irinotecan (FOLFOXIRI) compared with infusional fluorouracil, leucovorin, and irinotecan (FOLFIRI) as first-line treatment for metastatic colorectal cancer: The Gruppo Oncologico Nord Ovest, J Clin Oncol, 2007;25: 1670-76.

10. Alberts SR, Horvath WL, Sternfeld WC, et al., Oxaliplatin, fluorouracil, and leucovorin for patients with unresectable liveronly metastases from colorectal cancer: a North Central Cancer Treatment Group phase II study, J Clin Oncol, 2005;23:9243(9).

11. Kennedy AS, Nutting C, Coldwell D, et al., Pathologic response and microdosimetry of $(90) \mathrm{Y}$ microspheres in man: review of four explanted whole livers, Int I Radiat Oncol Biol Phys, 2004;60: 1552-63.

12. Gray B, Van Hazel G, Hope M, et al., Randomised trial of SIRSpheres plus chemotherapy vs. chemotherapy alone for treating patients with liver metastases from primary large bowel cancer, Ann Oncol, 2001;12:1711-20.

13. Van Hazel G, Blackwell A, Anderson J, et al., Randomised phase 2 trial of SIR-spheres plus fluorouracil/leucovorin chemotherapy versus fluorouraci//leucovorin chemotherapy alone in advanced colorectal cancer, I Surg Oncol, 2004;88:78-85.

14. Stubbs RS, Wickremesekera SK, Selective internal radiation therapy (SIRT): a new modality for treating patients with colorectal liver metastases, HPB, 2004;6:133-9.

15. Kennedy AS, Coldwell D, Nutting C, et al., Resin 90Y-microsphere brachytherapy for unresectable colorectal liver metastases: modern USA experience, Int J Radiat Oncol Biol Phys, 2006;65:412-25.

16. Sharma R, Van Hazel G, Morgan B, et al., Radioembolization of liver metastases from colorectal cancer using yttrium-90 microspheres with concomitant systemic oxaliplatin, fluorouracil, and leucovorin chemotherapy, J Clin Oncol, 2007;25:1099-1106.

17. Goldstein D, Van Hazel G, Pavlakis N, et al., Selective internal radiation therapy (SIRT) plus systemic chemotherapy with irinotecan. A phase I dose escalation study, ASCO Annual
Meeting Proceedings, J Clin Oncol, 2005; Abstract 3701.

18. Van Hazel $G$, Price $D$, Bower $G$, et al., Selective internal radiation therapy (SIRT) plus systemic chemotherapy with irinotecan. A phase I dose escalation study ASCO Annual Meeting GI Symposium, 2005; Hollywood, Florida. Abstract 108.

19. Van Hazel $G$, Internal radiation combined with modern chemotherapy, Liver Directed Radiotherapy with Microspheres: Second Annual Clinical Symposium April 2006

20. Giacchetti S, Perpoint B, Zidani R, et al., Phase III multicenter randomized trial of oxaliplatin added to chronomodulated fluorouracil-leucovorin as first-line treatment of metastatic colorectal cancer, J Clin Oncol, 2000;18:136-47.

21. de Gramont A, Figer A, Seymour M, et al., Leucovorin and fluorouracil with or without oxaliplatin as first-line treatment in advanced colorectal cancer, J Clin Oncol, 2000;18:2938-47.

22. Van Cutsem E, Blijham $G$, Irinotecan versus infusional 5-fluorouracil: a phase III study in metastatic colorectal cancer following failure on first line 5-fluorouracil, V302 Study Group, Semin Oncol, 1999;26:13-20.

23. Van Cutsem E, Dirix L, Van Laethem JL, et al., Optimisation of irinotecan dose in the treatment of patients with metastatic colorectal cancer after 5-FU failure: results from a multinational, randomised phase II study, Brit J Cancer, 2005;92:1055-62.

24. Schoemaker N, Kuppens IE, Moiseyenko V, et al., A Randomised phase II multicentre trial of irinotecan (CPT-11) using four different schedules in patients with metastatic colorectal cancer, Brit J Cancer, 2004;91:1434-41.

25. Weiss L, Grundmann E, Torhorst J, et al., Haematogenous metastatic patterns in colonic carcinoma: an analysis of 1541 necropsies, J Pathol, 1986; 150:195-203.

26. Folprecht $\mathrm{G}$, Grothey $A$, Alberts $S$, et al., Neoadjuvant treatment of unresectable colorectal liver metastases: correlation between tumour response and resection rates, Ann Oncol, 2005;16:1311-19.

27. Coldwell DM, Kennedy AS, Nutting CW, Use of yttrium-90 microspheres in the treatment of unresectable hepatic metastases from breast cancer, Int I Radiat Oncol Biol Phys, 2007; Epub: doi:10.1016/j.jijrobp.2007.03.056; May 2007.

28. Coldwell D, Nutting C, Kennedy A, Use of yttrium-90 SIRspheres to treat unresectable metastatic neuroendocrine tumors in the liver, World Congress of Gastrointestinal Cancer, 2005; Abstract 0-002.

29. King J, Morris D, Glenn D, et al., SIR-Spheres for liver metastases from neuroendocrine cancer, European Neuroendocrine Society (ENETS) meeting 2006; Abstract C28.

30. Kennedy A, Liu D, Dezarn AW, et al., Resin 90Y-microspheres for unresectable neuroendocrine hepatic metastases, Liver Directed Radiotherapy with Microspheres: Second Annual Clinical Symposium, 27-28 April 2006, Am I Oncol Review, 2006; 4(5 Suppl. 5):2.

31. Pöpperl $G$, Helmberger $T$, Munzing $W$, et al., Selective internal radiation therapy with SIR-Spheres in patients with nonresectable liver tumors, Cancer Biother Radiopharm, 2005:20(2):200-8.

32. Jakobs TF, Hoffmann RT, Poepperl $G$ et al., Mid-term results in otherwise treatment refractory primary or secondary liver confined tumours treated with selective internal radiation therapy (SIRT) using 90Yttrium resin-microspheres, European Radiology,
2007;17:1320-30

33. Szyszko T, AL-Nahhas A, Canelo R, et al., Assessment of response to treatment of unresectable liver tumours with $90 \mathrm{Y}$ microspheres: Value of FDG PET versus computed tomography, Nucl Med Commun, 2007;28:15-20.

34. Gulec SA, Mesoloras G, Dezarn WA, et al., Safety and efficacy of Y-90 microsphere treatment in patients with primary and metastatic liver cancer: The tumor selectivity of the treatment as a function of tumor to liver flow ratio, I Trans/ Med, 2007:5:15.

35. Lau WY, Leung WT, Ho S, et al., Treatment of inoperable hepatocellular carcinoma with intrahepatic arterial yttrium-90 microspheres: a phase I and II study, Br J Cancer, 1994;70:994-9.

36. Lau WY, Ho S, Leung TW, et al., Selective internal radiation therapy for nonresectable hepatocellular carcinoma with intraarterial infusion of 90-yttrium microspheres, Int J Radiat Oncol Biol Phys, 1998;40:583-92.

37. Lau WY, Ho S, Leung WT, et al., What determines survival duration in hepatocellular carcinoma treated with intraarterial Yttrium-90 microspheres?, Hepatogastroenterology, 2001;48:338-40.

38. Lau WY, Ho SK, Yu SC, et al., Salvage surgery following downstaging of unresectable hepatocellular carcinoma, Ann Surg, 2004;240:299-305.

39. Sangro B, Bilbao JI, Boan J, et al., Radioembolization using $90 \mathrm{Y}$ resin microspheres for patients with advanced hepatocellular carcinoma, Int J Radiat Oncol Biol Phys, 2006;66:792-800.

40. Coldwell D, Treatment of unresectable nodular cholangiocarcinoma with yttrium-90 microspheres, Ann Oncol, 2006 World Congress of Gastrointestinal Cancer; 17(Sup 6):vi56 P-102.

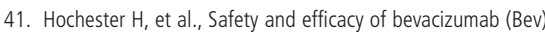
when added to oxaliplatin/fluoropyrimidine (O/F) regimens as first-line treatment of metastatic colorectal cancer (mCRC): TREE 1 \& 2 Studies, I Clin Oncol, 2005;23:249s (Abstract 3515).

42. Hochester $H$, et al., Safety and efficacy of oxaliplatin/fluoropyrimidine regimens with or without bevacizumab as first-line treatment of metastatic colorectal cancer (mCRC): Final analysis of the TREE-Study, J Clin Oncol, 2006; 24 (June 20 Suppl.): 148s (Abstract 3510).

43. Hurwitz H, Fehrenbacher L, Hainsworth JD, et al., Bevacizumab in combination with Fluorouracil and leucovorin: an active regimen for first-line metastatic colorectal cancer, ASCO Annual Meeting Proceedings, I Clin Oncol, 2005:23:3502-8.

44. Kopetz S Abruzzese JL, Eng C, et al., Preliminary results from a phase II study of infusional 5-FU, leucovorin, and irinotecan (FOLFIRI) plus bevacizumab as first-line treatment for metastatic colorectal cancer (mCRC), ASCO Annual Meeting Proceedings Part I, J Clin Oncol, 2006;24, No 185 (Supplement): (Abstract 3579).

45. Dittrich C, Hoehler T Lordick F, et al., A phase I/II study of cetuximab in combination with 5-fluorouracil (5-FU)/folinic acid (FA) plus weekly oxaliplatin (L-OHP) (FUFOX) in the first-line treatment of patients with metastatic colorectal cancer (mCRC) expressing epidermal growth factor receptor (EGFR), preliminary results, World Congress of Gastrointestinal Cancer, Ann Oncol, 2006;17 (Sup 6): vi24 Abstract 0-019. 


\section{QuadraSphere \\ EXPANDING MICROSPHERES}

\section{The first ACTIVE embolic provides $4 X$ the Benefits for Effective Embolization}

\section{QuadraSphere ${ }^{\mathrm{Tm}}$ Microspheres enable targeted occlusion for effective embolization in four important ways:}

\section{Targeted}

Retains spherical shape with consistent cross sectional diameter after the reconstitution with aqueous-based solutions such as contrast media and $0.9 \%$ saline solution for predictable flow-directed level of occlusion in the vasculature.

\section{Absorbing}

Rapidly absorbs contrast media and $0.9 \%$ saline solution. In addition to the absorption process, the negatively charged polymer is also capable of selective bonding with positively charged material through an ionic interaction process. These technology attributes provide a unique and versatile embolization platform.

\section{Conforming}

Affords atraumatic conformability to the architecture of the vessel lumen, providing more contact surface area with the embolic material and the vessel intima, lending to a more complete occlusion of the vessel. In vivo studies demonstrate the ability for one sphere to occlude bifurcated vessel branches.

\section{Expanding}

Expansion up to four times the stated dry diameter with secondary conformability to the vessel architecture, resulting in an increase in surface area contact for a more complete vessel occlusion.

For more information, please visit www.biospheremed.com or call 800-394-0295. 